\title{
Quantitative Imaging of Temperature Elevations in Tissues Due to Thermal Therapies
}

\author{
Jeremy P. Kemmerer, Goutam Ghoshal, Michael L. \\ Oelze \\ Department of Electrical and Computer Engineering \\ University of Illinois at Urbana-Champaign \\ Urbana, IL, USA \\ oelze@illinois.edu
}

\author{
Nicholas Rubert, Kayvan Samimi \\ Department of Medical Physics \\ University of Wisconsin \\ Madison, WS, USA
}

\begin{abstract}
Thermal ablation and hyperthermia remain as potent treatment options for cancer. However, the inability to closely monitor temperature elevations from thermal therapies in real time continues to limit clinical applicability. Therefore, the development of new imaging techniques capable of providing feedback and temperature monitoring is highly medically significant. In this study, quantitative ultrasound imaging techniques based on spectral estimates were examined for their ability to monitor and map temperature elevations induced in tissues using either microwave ablation or high intensity focused ultrasound (HIFU).
\end{abstract}

Ex vivo liver samples were treated with microwave ablation while ultrasound image frames were recorded using a SonixTouch system and linear array (14L5). In vivo tumors in rats (MAT) were treated using a custom-built HIFU system while concurrently imaged using a SonixRP scanner and linear array (14L5). The ultrasound scanners provided raw radio frequency (RF) data. From the RF data, the backscatter coefficient was calculated using the reference phantom technique. The backscatter coefficient was parameterized by estimating an effective scatterer diameter (ESD) and effective acoustic concentration (EAC) assuming a spherical Gaussian model. Maps of the ESD and EAC were created for each acquired frame. Temperature was measured by placing a needle thermocouple in the samples during treatment and temperature changes were correlated with changes in the scattering parameters.

In the ex vivo liver samples, the ESD was observed to increase with temperature elevation while the EAC was observed to decrease with temperature elevation. Specifically, the mean ESD increased by $7 \mu \mathrm{m}$ and EAC decreased by $1.5 \mathrm{~dB}$ as the temperature increased from 18 to $42{ }^{\circ} \mathrm{C}$. Conversely, in the in vivo tumor samples treated with HIFU, the EAC was observed to increase with increasing temperature, i.e., the EAC increased by 20 to $30 \%$ as the temperature increased from $37{ }^{\circ} \mathrm{C}$ to a range of 50 to $60{ }^{\circ} \mathrm{C}$. When the HIFU was turned off, the EAC continued to track the decrease in temperature of the tumor. In the in vivo studies, the tumors were grown on the chest wall of the rats and, therefore, large out of plane tissue motion occurred due to the breathing of the animal. In spite of this, the EAC parameter was capable of tracking temperature in the presence of large tissue motion.

Keywords-Thermometry; Quantitative ultrasound; HIFU; Backscatter coefficient

\section{INTRODUCTION}

Hyperthermia and thermal ablation techniques remain important options for the treatment of several different diseases. The most commonly used thermal treatments include RF ablation and microwave ablation. However, high-intensity focused ultrasound (HIFU) remains a promising technique for clinical therapies involving non-invasive thermal ablation or hyperthermia [1]. Recent applications of HIFU include ablation of cancerous tumors, treatment of bone metastases, and uterine fibroids. HIFU can target small regions of tissue noninvasively for thermal ablation or hyperthermia treatment and has been successfully demonstrated in animals and in clinical studies and treatments. A review of clinical studies involving HIFU to treat disease was provided by Kennedy [1]. Currently in the USA, HIFU therapy is only approved to treat uterine fibroids and bone metastases.

One continuing issue with thermal therapy techniques is the inability to map temperature changes in tissues in real time and with high accuracy. Currently, MRI is considered the gold standard for mapping temperature changes in tissues during thermal therapies. An accuracy of $1^{\circ} \mathrm{C}$ and 1 second with spatial resolution of approximately $2 \mathrm{~mm}$ has been reported for MRI thermometry for non-moving tissue [2]. When motion is present, proton-resonance frequency techniques for MRI thermometry have been explored but have achieved only nearreal time (i.e., $10 \mathrm{~Hz}$ ) and require predictable motion patterns to implement the registration algorithms [2-5]. However, MRI is an expensive imaging modality, non-portable and requires specialized ablation equipment in order not to interfere with the MRI system.

Ultrasound is an attractive imaging modality to guide and monitor HIFU treatment because it is non-ionizing, inexpensive, portable and capable of real-time imaging. Because of its attractiveness, several ultrasonic techniques have been investigated for monitoring, quantifying, and mapping the temperature rises induced in tissues by HIFU treatment. The most widely explored approach to ultrasonic thermometry is to quantify the changes in speed of sound and/or attenuation [69]. However, these techniques are susceptible to tissue motion and depend on the specific tissue properties to deduce temperature changes from changes in sound speed or attenuation. Furthermore, changes in sound speed or

This work was supported by a grant from the NIH, R01 EB008992. 
attenuation are not monotonic. In attempts to overcome motion artifacts, researchers have developed fast frame rate techniques [9].

In this study, the use of spectral domain information from ultrasound backscattered signals was examined for its ability to track temperature changes in in vivo and ex vivo tissues. The hypothesis being tested was that scattering properties of tissues change when undergoing temperature elevations and these changes can be used to track temperature.

\section{MethodS}

\section{A. Animal Procedures}

The experimental protocol was approved by the Institutional Animal Care and Use Committee (IACUC), University of Illinois, Urbana-Champaign and satisfied all University and NIH rules for the humane use of laboratory animals. A rat mammary adenocarcinoma cell line, 13762 MAT B III (MAT) cells (American Type Culture Collection (ATCC), Manassas, Virginia, USA) were cultured in ATCCformulated McCoy's 5a Medium Modified along with 10\% fetal bovine serum (Hyclone Laboratories, Logan, Utah, USA) and $1 \%$ penicillin-streptomycin (Hyclone Laboratories, Logan, Utah, USA) under conditions of $5 \% \mathrm{CO}_{2}$ and $95 \%$ humidity at $37{ }^{\circ} \mathrm{C}$. A Reichert Bright-Line ${ }^{\circledR}$ hemocytometer (Hausser Scientific, Buffalo, New York, USA) was used to count viable cells and estimate the concentration of cells. A volume of 100 $\mu \mathrm{L}$ containing 500 cells was injected subcutaneously into both the right and left abdominal mammary fat pads of female 10-21 week-old Fischer 344 rats (Harlan Laboratories, Inc, Indianapolis, IN). Prior to injecting the MAT cells, the rats were weighed and then anesthetized with isoflurane. Ultrasonic experiments were conducted on animals that had tumors of 8-9 $\mathrm{mm}$ in diameter. For each experiment, hair over the tumor was shaved and depilated before the ultrasound exposure. Twelve rats were used in the experiments.

For ex vivo exposures, fresh bovine liver samples were acquired from butchered animals. The samples were refrigerated until used in experiments. Three liver samples were used in the experiments.

\section{B. Ultrasound Exposures}

The MAT tumors in the rats were exposed in vivo to HIFU using a single-element $1-\mathrm{MHz}$ (f/1.1) transducer with aperture diameter of $4.7 \mathrm{~cm}$ that was powered by an A150-55 dB power amplifier (ENI, Rochester, NY) and excited by an arbitrary waveform generator (HP 33120a, Agilent Technologies, Santa Clara, CA). The $(-6 \mathrm{~dB})$ beamwidth and focal length of the HIFU transducer was $1.8 \mathrm{~mm}$ and $5.2 \mathrm{~cm}$, respectively as measured in degassed water at $37{ }^{\circ} \mathrm{C}$ using needle hydrophone (Precision Acoustics, Dorchester, UK). The exposure was monitored using a SonixRP clinical ultrasound system (Ultrasonix, Richmond, BC, Canada) and an L14-5/38 probe with signals recorded at a sampling frequency of $40 \mathrm{MHz}$. The probe had a center frequency of 7.2 $\mathrm{MHz}$ as estimated in pulse-echo mode. A custom holder was designed to hold both the HIFU transducer and imaging probe such that the focal region of the HIFU transducer was aligned automatically with the imaging plane of the SonixRP probe and no further adjustment was required. The experimental setup is shown in Fig. 1. A needle thermocouple (HYP1-301/2-T-G-60-SMPW-M, Omega Engineering, Inc., Stamford, Connecticut) was inserted into the tumor to monitor the temperature profile during the experiment. The diameter and the length of the needle thermocouple were $0.3 \mathrm{~mm}$ and 15 $\mathrm{mm}$, respectively. The thermocouple was connected to a temperature reader (NI USB-TC01, National Instruments Corporation, Austin, Texas), which was connected to a computer to record temperature every one second. The degassed water bath was maintained at $37{ }^{\circ} \mathrm{C}$ using an automatic temperature controller throughout the ultrasonic experiment. In an experiment, the sample was moved using a computer-controlled micro-positioning system (Daedal, Inc., Harrisburg, PA). Reference spectra were acquired by scanning a well-characterized tissue-mimicking phantom with the same equipment settings.

For ex vivo exposures, a Certus 140 PR microwave ablation probe (Neuwave Medical, Madison, WI) was inserted into the liver sample and used to induce temperature elevations in the liver samples. A SonixTouch (Ultrasonix, Richmond, BC, Canada) was used to image the location of the ablation probe tip in the liver sample. The probe was then fixed in this location and RF data were recorded for 5 minutes as the microwave ablation device was used to induce temperature elevations in the liver sample. Reference spectra were acquired by scanning a well-characterized tissuemimicking phantom with the same equipment settings.

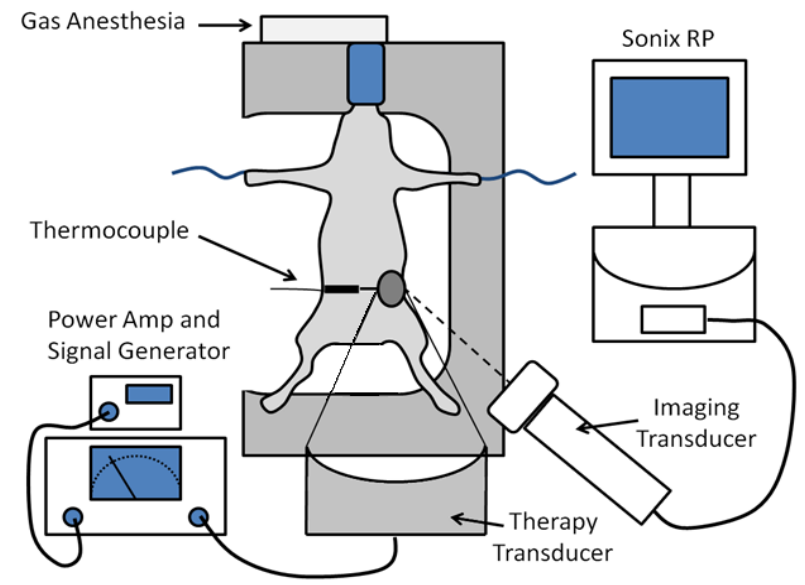

Figure 1. Experimental setup for therapeutic ultrasound exposure.

\section{Estimation of Scattering Properties.}

A region of interest (ROI) containing the tumor or the region around the ablation probe was outlined from B-mode images constructed from the RF data. The ROI was broken into smaller data blocks of $3 \mathrm{~mm}$ by $3 \mathrm{~mm}$. The backscatter coefficient (BSC) was estimated from RF data in each data block. Estimates of the effective scatterer diameter (ESD) and effective acoustic concentration (EAC) were obtained from the BSC estimate using a spherical Gaussian scattering model [10]. Maps of parameter estimates were constructed at each 
time point by relating the data block to a pixel and color coding with the parameter value (ESD or EAC). Data blocks had a $75 \%$ overlap with one another; therefore, the scatterer property values associated with each pixel was an average of several data block estimates.

\section{RESULTS}

\section{A. HIFU Exposures}

Figure 2 shows QUS images (both ESD and EAC) of a representative rat tumor at different time points during HIFU exposure and after HIFU exposure. To the right of the images are the associated temperature curves and QUS parameter curves. The QUS parameter curves were generated by averaging the QUS parameters over the whole ROI and calculating the percent difference between the QUS parameter at each time point with the QUS parameter average at baseline, i.e., before HIFU was turned on. Examination of the images reveals that significant motion occurred during the HIFU exposure because the rat was alive and breathing. Because the rat is a small animal and the tumor was on the chest wall of the rat, large motion out of plane and both laterally and vertically were observed. The jagged nature of the QUS parameters as the temperature increased is hypothesized to be due in part to the motion of the tumor during exposure. In almost all animals, the EAC was observed to increase with temperature and decrease once the temperature began to decrease.

\section{B. Microwave Ablation}

Figure 3 shows graphs of changes in QUS parameters versus time of exposure. Plotted along with the QUS parameters is the temperature curve from the thermocouple. QUS parameters represent an average of QUS parameters over the ROI drawn around the microwave probe tip. In the liver sample experiments, no motion was present and variations in QUS parameter estimates were smaller than in the corresponding rat tumors. Contrary to the HIFU exposures in the tumor samples, the EAC decreased with increasing temperature while the ESD increased with increasing
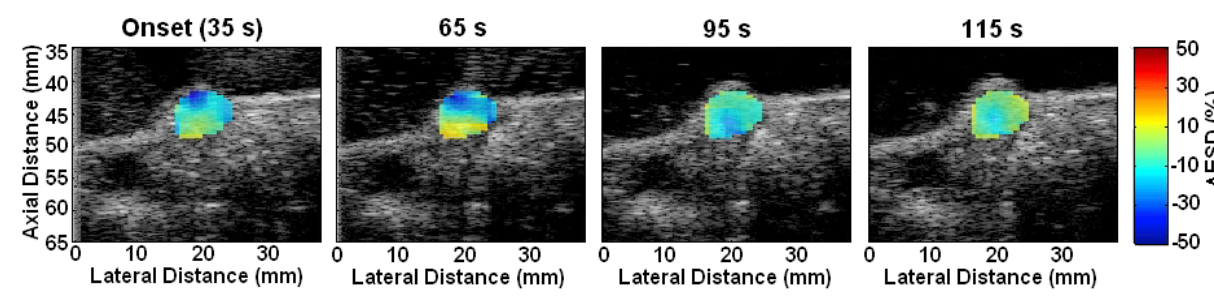

\section{DiscusSION}

The images and data suggest that underlying scatterer properties are changing relative to background as the temperature of the tissue changes. In tumors the EAC was observed to increase with temperature in almost every case while the ESD was observed to decrease in a majority of cases. For the ex vivo liver samples, the opposite occurred. Namely, the EAC decreased with increasing temperature and the ESD increased with increasing temperature. We observed similar characteristics in changes in QUS parameters with HIFU

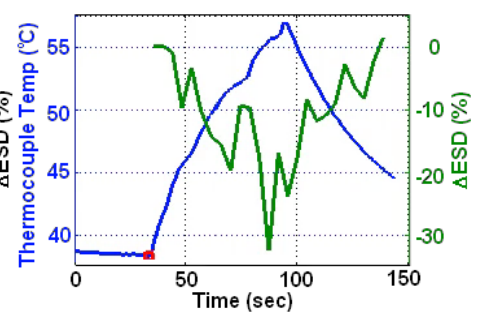

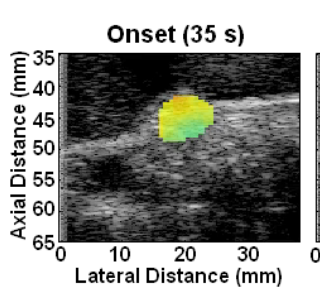
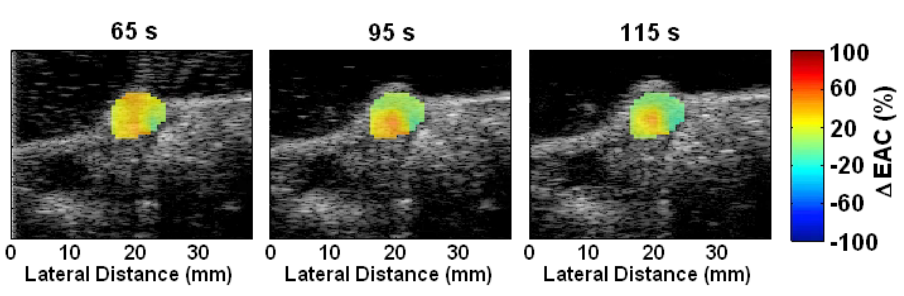

Figure 2. (Top) Series of ESD difference images of tumor during treatment with HIFU and corresponding temperature/ESD curves versus time. (Bottom) Series of EAC difference images of tumor during treatment with HIFU and corresponding temperature/EAC curves versus time. 
exposures in freshly excised bovine liver samples in our previous studies [11]. The differences in the two cases for the current study could be due to different tissue structures or to different exposure conditions. Under the hypothesis that the main effect of both exposure techniques is to elevate the temperature, then the differences in observed effects would be due to different tissue structures. However, more studies are required to determine if the differences in observed effects are due to different tissue structures or exposure conditions.

Another observation is that the QUS curves versus
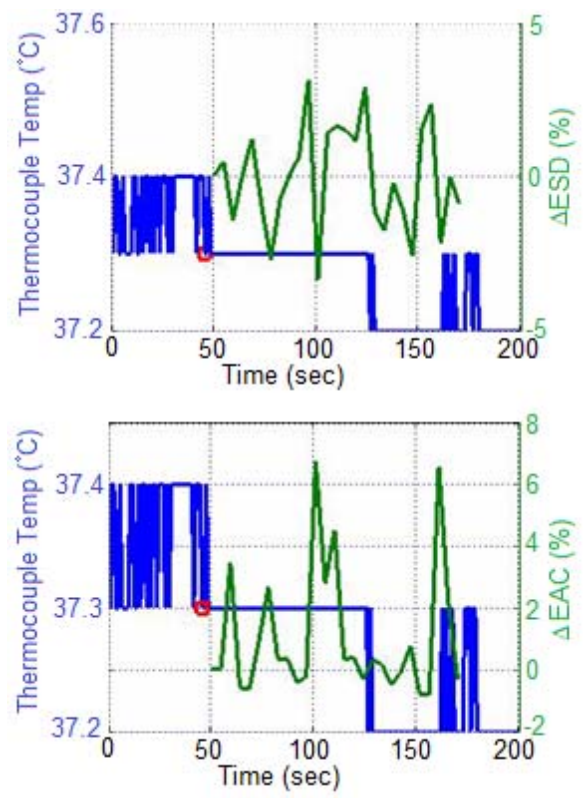

Figure 4. (Top) Changes in ESD versus time and (bottom) changes in EAC versus time from rat tumor with no HIFU.

exposure time are not as smooth as the corresponding temperature curves. Part of this effect was hypothesized to be due to motion of the underlying sample. Comparing the in vivo data to the ex vivo data showed much larger variations in the QUS curves for the in vivo data. To further elucidate these effects RF data were recorded from a rat tumor over time without HIFU being turned on. Figure 4 shows a graph of curves generated with changes in QUS parameters due to motion of the rat tumor. With no HIFU exposure, no trends in the QUS parameters were observed; however, small fluctuations were observed in the QUS parameters as time elapsed. These variations are mainly due to motion of the tumor during imaging and would partially account for some of the observed fluctuations observed in the QUS images created during and after HIFU exposure. On the other hand, the fact that the QUS could maintain the correspondence with the temperature trend in the presence of large amounts of tissue motion including both in plane and out of plane motion suggests that QUS imaging will be more robust against tissue motion. Averaging of frames in slow time could further alleviate the jumpiness of QUS thermometry over time.

QUS imaging was used to correlate changes in tissue scattering properties to temperature changes in tissue due to therapy. QUS parameters were observed to change with respect to both increases and decreases in temperature in tissues. Therefore, QUS could be an ultrasound-based alternative to thermometry because it tracks temperature and appears to be robust against tissue motion. The curves generated for the QUS parameters were from averages of QUS parameters over larger ROIs. Therefore, the spatial resolution of temperature imaging with QUS was not quantified. The performance of the QUS imaging for temperature tracking may be improved if the QUS maps could be more closely correlated in space with actual temperature maps. Finally, more studies need to be conducted to determine the temperature resolution of the QUS imaging technique and to determine the tissue dependencies of QUS for temperature monitoring.

\section{ACKNOWLEDGMENT}

The authors would like to thank Professor William D. O'Brien, Professor Tomy Varghese, and Dr. Rita Miller for their help with interpretations of results and their technical assistance with the experiments.

\section{REFERENCES}

[1] J. E. Kennedy, "High-intensity focused ultrasound in the treatment of solid tumours," Nature reviews. Cancer, 5, 321-7, 2005.

[2] B. Denis de Senneville, B. Quesson, and C. T. Moonen, "Magnetic resonance temperature imaging," Intern J Hypertherm, 21, 515-31, 2005.

[3] M. Ries, B. D. De Senneville, S. Roujol, S. Hey, G. Maclair, M. O. Kohler, B. Quesson, and C. T. W. Moonen, "Three Dimensional Motion Compensation for Real-Time MRI Guided Focused Ultrasound Treatment of Abdominal Organs," AIP Conference Proceedings, 1215, 239-242, 2010.

[4] B. Quesson, C. Laurent, G. Maclair, B. Denis de Senneville, C. Mougenot, M. Ries, T. Carteret, A. Rullier, and C. T. W. Moonen, "Real-time volumetric MRI thermometry of focused ultrasound ablation in vivo: a feasibility study in pig liver and kidney," NMR in biomedicine, 24, 145-153, 2011.

[5] M. Ries, B. D. de Senneville, Y. Regard, and C. Moonen, "Combined magnetic resonance imaging and ultrasound echography guidance for motion compensated HIFU interventions," AIP Conference Proceedings, 1503, 202-206, 2012.

[6] A. Anand, D. Savery, and C. Hall, "3-D spatial and temporal temperature imaging in gel phantom using backscattered ultrasound," IEEE Trans Ultrason. Ferroelectr. Freq. Control, 54, 23-31, 2007.

[7] M. J. Daniels, T. Varghese, E. L. Madsen, and J. A. Zagzebski, "Noninvasive ultrasound-based temperature imaging for monitoring radiofrequency heating-phantom results," Physics Med Biol, 52, 482743, 2007.

[8] C. Simon, P. Vanbaren, and E. S. Ebbini, "Two-dimensional temperature estimation using diagnostic ultrasound," IEEE Trans Ultrason. Ferroelectr. Freq. Control, 45, 1088-99, 1998.

[9] D. Liu and E. S. Ebbini, "Real-time 2-D temperature imaging using ultrasound," IEEE Trans BioEng, vol. 57, pp. 12-6, Jan 2010.

[10] M. L. Oelze, J. F. Zachary, and W. D. O'Brien, Jr., "Characterization of tissue microstructure using ultrasonic backscatter: theory and technique for optimization using a Gaussian form factor," J Acoust Soc Amer, 112, 1202-11, 2002.

[11] G. Ghoshal, A. C. Luchies, J. P. Blue and M. L. Oelze, "Temperature dependent ultrasonic characterization of biological media," J Acoust Soc Amer, 130, 2203-2211, 2011. 